\title{
Téoros
}

Revue de recherche en tourisme

\section{Le patrimoine industriel : une richesse de notre environnement}

\section{Jacques Lecours}

Volume 10, numéro 1, mars 1991

Tourisme et environnement

URI : https://id.erudit.org/iderudit/1079819ar

DOI : https://doi.org/10.7202/1079819ar

Aller au sommaire du numéro

Éditeur(s)

Université du Québec à Montréal

ISSN

0712-8657 (imprimé)

1923-2705 (numérique)

Découvrir la revue

Citer cet article

Lecours, J. (1991). Le patrimoine industriel : une richesse de notre

environnement. Téoros, 10(1), 39-41. https://doi.org/10.7202/1079819ar d'utilisation que vous pouvez consulter en ligne.

https://apropos.erudit.org/fr/usagers/politique-dutilisation/ 


\section{Le patrimoine industriel: une richesse de notre environnement}

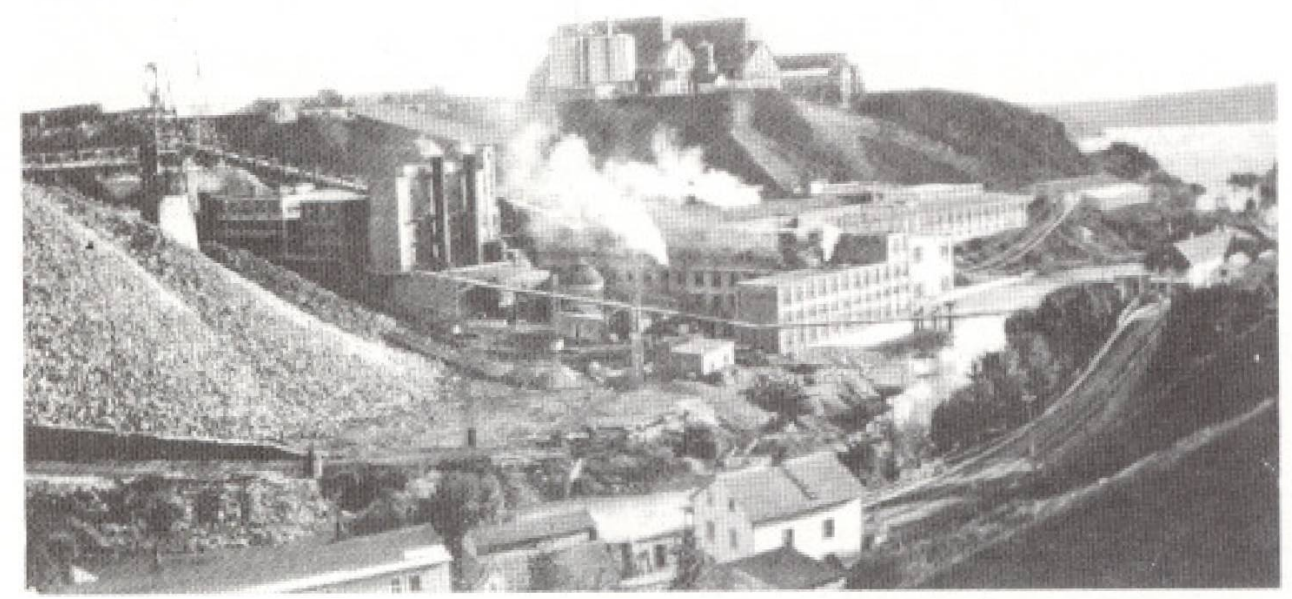

Si le secteur tertiaire est le fondement actuel des économies occidentales, il reste que ces économies sont directement issues de l'industrie. C'est elle qui a marquế non seulement notre développement économique, mais aussi notre physionomie collective, nos mentalités, notre paysage urbain, notre structure régionale, notre environnement biophysique.

La compréhension et l'appréciation d'un pays "industrialisé" ne peuvent être complètes sans ces rếalités. Aussi les sites industriels anciens sont-ils un instrument privilégié de contact avec des phénomènes souvent méconnus: la ville industrielle, l'impact de l'industrie sur le milieu naturel, le rốle bouleversant qu'a joué la technologie, les transferts de technologie, l'histoire ouvrière.

\section{Le patrimoine industriel dans le monde}

L'Angleterre, le berceau de l'ère industrielle, a été la première à reconnaître la signification historique de ses installations industrielles anciennes et la première à se

\footnotetext{
- Monsieur Jacques Lecours est president de
} I'Asscociaticn québecoisepour le patrimoine industriel préoccuper de leur sauvegarde et de leur mise en valeur. Ce pays est le lieu de naissance del'archéologie industrielle, une pratique qui fait appel à l'histoire de la science et des technologies et à l'histoire sociale mais qui s'attarde d'abord aux manifestations physiques de l'industrie, c'est-à-dire aux installations et à l'appareillage, en activité ou non. Plusieurs sites industriels anciens du nord de l'Angleterre, notamment dans les régions de Birmingham, de Manchester et de Leeds, sont devenus des sites touristiques axés sur l'histoire industrielle. On y trouve des sites qui mettent en valeur les grands secteurs d'activités de la révolution industrielle, comme les mines et les carrières, le charbon et la vapeur, les aciéries, les poteries, les filatures, les transports. Le plus connu de dans le Shropshire.

Tous les pays ne sont pas aussiavancés àcet égard mais on retrouve à peu près dans tous les paysqui ont une histoire industrielle des initiatives réussies qui démontrent le potentiel de sites industriels anciens comme éléments de l'infrastructure touristique. Il y a des sites dans tous les pays d'Europe, en Amérique du Sud, en Australie, au Japon. Les spécialistes de tous ces pays se réunissent régulièrementsousl'égide du TICCIH (The International Committee for The ces sites est le Ironbridge Gorge Museum
Conservation of The Industrial Heritage). Cette organisation tiendra son congrès à Montréal, Toronto et Ottawa en 1994.

Il $\mathrm{y}$ a aussi plusieurs sites touristiques qui mettent en valeur le patrimoine industriel aux États-Unis. Le plus célèbre et celui dont le développement est le plus avancé est Lowell, Mass, une ville de filatures qui a été un des foyers de la révolution industrielle américaine. Dès le début du XIXe siècle, un imposant réseau de canaux alimentait les turbines qui actionnaient les métiers de Lowell. Les filatures du nord-est des États-Unis connurent leur apogée au lendemain de la Première Guerre mondiale, apogée qui fut suivie par un déclin constant jusqu'à la fermeture des dernières usines dans les années 1960. Le site est devenu le Lowell National Historical Park, le premier parc national américain en milieu urbain. Les trois paliers de gouvernement se sont engagés dans la renaissance de la ville en remettant en état son patrimoine urbain, industriel et technologique. Aujourd'hui, lavilleconnaît unessor remarquable, non seulement à cause de sa nouvelle vocation touristique mais aussi parce qu'elle a su attirer des entreprises de haute technologie (Wang, le grand fabricant d'ordinateurs, notamment) dont certaines se sont installées dans les anciens bâtiments des filatures, particulièrement propices à la conversion à de nouveaux usages.

On trouvera partout aux États-Unis des sites industriels anciens. Tout près de nous, on peut visiter, outre Lowell, Saugus Iron Works dans le Massachussetts, le Museum of Textile History au New Hampshire, Slater Historic Mill au Rhode Island, le Bath Maritime Museum dans le Maine. Ce ne sont là que quelques exemples et on peut mieux connaître ce patrimoine par l'entremise de la Society for Industrial Archeology. Cet organisme a d'ailleurs tenu son congrès annuel 1989 à Québec à l'invitation de la Commission des biens culturels du Québec, et les congressistes américains ont été impressionnés par la diversité et la richesse de notre patrimoine industriel.

AuCanadaégalement, plusieurs sites industriels historiques ont fait l'objet d'une mise en valeur touristique. Parmi les projets les plus avancés, mentionnons le "Steel Project" 
qui présente l'histoire de l'aciérie à l'île du Cap-Breton en Nouvelle-Écosse, le Musée et les Archives Seagram à Waterloo, Ontario, qui explique l'histoire de l'industrie de la distillerie et, enfin, des sites d'anciennes conserveries de saumon en Colombie-Britannique.

Au Québec, la diversité des sites présentement à l'étude et le succès des sites qui sont déjà ouverts aux visiteurs indiquent que les installations industrielles anciennes peuvent devenir une composante importante de l'infrastructure touristique de nos régions. Ces sites ont été développés ou sont en voie de développement sous l'égide d'une variété étonnante d'acteurs: grandes entreprises, gouvernements et municipalités, sociếtés d'histoire et groupes de citoyens. Un répertoire récemment préparé par $1^{\prime}$ Association québécoise pour le patrimoine industriel dénombre au-delà de cent cinquante personnes qui se consacrent exclusivement ou principalement à la connaissance, la protection et la mise en valeur du patrimoine industriel. C'est que la matière ne manque pas!

\section{Le rôle historique de l'eau dans le développement industriel du Québec}

L'eau occupe près de dix-huit pour cent de la superficie du Québec. Les cours d'eau y ont joué un rôle fondamental en permettant le transport et les communications des le début de la colonisation. L'eau a aussi donné naissance à une importante industrie de construction navale, d'abord à l'époque du bois, puis à l'ère de l'acier. L'eau a été utilisée dans les procédés mêmes de transformation et de fabrication pour décomposer, pour nettoyer, pour refroidir ou... pour transporter les déchets.

La puissance de l'eau a toujours été exploitée, d'abord dans de nombreux moulins et ensuite dans les centrales hydroélectriques qui caractérisent le Québec. Plusieurs moulinsde l'époque seigneuriale et duXIXe siècle ont été préservés, restaurés et mis en valeur, parexempleà Saint-Eustache(DeuxMontagnes), à Beaumont(Bellechasse) et à Saint-Roch-des-Aulnaies(L'Islet). A Terrebonne, sur l'île des Moulins, il y a un ensemble remarquable de moulins dont les plus anciens datent de 1721 . Il y a eu à cet endroit des moulins à scie, des moulins à farine et des moulins à carder. Le ministère des Affaires culturelles du Québec y a fait d'importants travaux de restauration depuis une quinzaine d'années.

La première utilisation véritablementindustrielle, c'est-à-dire mécanisée, de la puis- sance hydraulique a ếté dans l'industrie du textile. Le Moulin d'Ulverton(Drummond) par exemple, a été construit à la fin des années 1860 . Il utilise des machines actionnées par l"eau pour transformer la laine en fil à tisser pour métiers mécaniques. On y produira ensuite des pièces tissées, des écheveaux de laine et du fil bobiné pour le commerce. C'est un site passionnant où l'on peut encore voir des vieilles machines en marche: cardeuse, fileuse, dévidoir, bobineuse, doubleuse. Le Moulin d'Ulverton, seul survivant de son espece (entre l'artisanat et la grande industrie) a été rescapé grâce au travail decitoyens de la région, maintenant appuyés par l'État.

Le sort des vestiges de la grande industrie textile du Québec est, quant à lui, très variable. Les beaux grands bâtiments de la Penman àSaint-Hyacinthe et de la Wabasso à Trois-Rivières ont été complètement rasés ces dernières années, sans qu'on ait sérieusement évalué leur potentiel de réutilisation. Pourtant, si on pense à l'exemple de Lowell, ce potentiel est véritable. Au centre-ville de Sherbrooke, les usines de la Paton ont été converties en logements, commerces et bureaux. Une grande partie des filatures de la Dominion Textile aux chutes Montmorency est disparue et ce qui subsiste sera converti en hôte et condominiums. Pourtant, il y aurait eu là l'occasion de présenter le passé industriel de la région au vaste auditoire touristique de la côte de Beaupré.

Toutes ces grandes filatures, très semblables d'ailleurs à celles de la NouvelleAngleterre, se sont installées sur des cours d'eau dont elles utilisaient la puissance. Dès la fin du siècle passé, les turbines ont été couplées non plus directement aux machines mais plutôt à des générateurs électriques. Cette nouvelle puissance transportable a révolutionné la physionomie industrielle en permettant dès lors aux usines de se localiser près de leur maind'oeuvre ou de leurs matières premières. C'est l'ère de l'hydroélectricité.

Le patrimoine hydroélectrique du Québec est facile d'accès aux touristes et plusieurs projets actuellement en préparation le rendront présent dans toutes les régions. Les grandescentralesd'Hydro-Québec sont déjà accessibles an public: Rivière-des-Prairies, Carillon et Beauharnois dans la région de Montréal, Shawinigan en Mauricie, Manicouagan sur la Cote-Nord. Alcan ouvre aussi au public certaines de ses grandescentrales du Saguenay-Lac-Saint-Jean. Les petites installations anciennes sont aussi trè̀ intéressantes. C'est ainsi que le très beau site de la centrale Sept-Chutesà SaintFéréol-les-Neiges, une installation de 1916 pour l'instant désaffectée, est devenu en
1987 un lieu d'interprétation et de plein air très fréquenté, autant par les résidents des environs que par les touristes. C'est un site hydroélectrique typique ou se rejoignent la technologie et la richesse du milieu naturel. Il y a possibilité de créer des sites semblables à Baie-Comeau, Chicoutimi, Rivièredu-Loup, Coaticook, Sherbrooke et Hull ainsi qu'en plusieurs autres endroits de la vallée du Saint-Laurent ou subsistent des vestiges trè̀s intéressants des premières usines hydró́lectriques.

Mais le projet le plus ambitieux est sans contredit la création d'un Centre de l'interprétation del'industrieàShawinigan, le berceau de l'industrie lourde québécoise. C'est là qu'est née notre grande industrie hydrólectrique, notre papeterie, notreélectrochimie. Le potentiel touristique est indéniable et les responsables du projet mettent actuellement la dernière main au programme de développement, s'appuyant sur des études de rentabilité encourageantes.

\section{Les mines et la forêt}

Si la grande hydroélectricité s'est implantée au Québec, grâce à d'importants capitaux étrangers, c'est en grande partie pour tirer profit des richesses naturelles de ses diverses régions.

Le sol avait été exploité dès les débuts de la colonie. Le parc des Forges du SaintMauricequ'a développé le Service des Parcs d'Environnement Canada est un témoin important d'un siècle et demi de production sidérurgique depuis le Régime français. La Corporation pour le patrimoine sidérurgique de la Mauricie prépare actuellement un projetattrayant decircuitd'interprétation qui couvrirasix sites de forges, d'extraction et de fabrication du charbon de bois, le plus ancien datant de 1798 . Il s'agit bien de l'époque de la "proto-industrialisation", c'est-à-dire de l'industrie artisanale qui a précédé l'industrie mécanisée.

L'époque plus récente de l'industrie minière nous est présentée en Abitibi par le village minier de Boulamaque, un site historique à Val-d'Or classé en vertu de la Loi sur les biens culturels et par le Musée des mines de Malartic, une initiative de miniers retraités. Dans les Bois-Francs, des initiatives de conservation d'archives donneront sûrement naissanceà des projets de mise en valeur des saisissants vestiges de l'industrie del'amiante, un volet important del'histoire industrielle et ouvrière du Québec.

La forêt est aussi exploitée depuis longtemps en ce pays. Il reste un peu partout des vestiges de moulins à scie artisanaux, mais 
peu de témoignages physiques (beaucoup d'archives et de documents iconographiques cependant) des débuts de l'industrie du bois. On pourra visiter avec intérêt le Musée du bûcheron à Grandes-Piles en Mauricie, qui présente la vie et la culture matérielle des anciens travailleurs forestiers, ainsi que le poste Opémican sur le lac Témiscamingue pour comprendre $1^{\prime}$ industrie du flottage du bois.

C'est au Saguenay et au Lac-St-Jean que l'on pourra visiter des anciennes pulperies. La Pulperie de Chicoutimi a êté construite entre 1897 et 1923 par J.-E.-A. Dubuc, un personnage bien connu des Saguenayens. Cetensemble impressionnant de bâtiments etd'installations a été désaffecté en 1930et estdevenu depuis dix ans un site récrétatifet culturel qui reçoit presque 200000 personnes par année. La Pulperie est maintenant le moteur principal du développement touristique de Chicoutimi. Les projets de développement du site prévoient un important volet d'interprétation des industries de la forêt, malgré la disparition de presque tout l'appareillage de la vieille Pulperie. À Val-Jalbert, une Pulperie au Lac-St-Jean, il $y$ a encore des vestiges des machines. ValJalbert a été très fréquenté des touristes depuis de nombreuses années, avant même sa mise en valeur. Tout comme SeptChutes, $c^{*} e s t$ un ensemble industriel ancien complet avec son propre village. Un endroit fascinant.

A Saint-Jérôme, il y a les vestiges de la Pulperie Wilson. Ils seront intégrés au réseau d'interprétation du parc de la Rivière du Nord. A Rivière-du-Loup, il y a une Pulperie artisanale, la Mohawk, qui était encore en opération voici quelques années. Tout l'appareillage est encore en place. Le site n'est pas ouvert aux visiteurs, mais il faut espérer qu'il le sera bientôt.

Le Service canadien des parcs a évalué la richesse patrimoniale d'une quinzaine de grandes usines papetières au Canada, dont plusieurs au Québec. La Commission des lieux et monuments historiques du Canada s'intéresse à cette industrie et juge qu'il est important d'en rappeler l'évolution. Une de ces usines sera peut-être ouverte aux visiteurs d'ici quelques années.

\section{Les manufactures et la ville ouvrière}

Tous ces sites rappellent le développement des diverses régions du Québec. Mais il ne faut pas oublier que c'est en milieu urbain qu'est née l'industrie mécanisée faisant appel à une nombreuse main-d'oeuvre: chaussuresà Québec, matériel ferroviaire à Montréal par exemple. À l'inverse, n'oublions pas qu'au tournant du siècle, les manufactures ont causé la transformation physique et sociale de nos villes. En s' implantant, elles ont provoqué le développement des grandes infrastructures urbaines: voies ferrées et routes, installations portuaires et canaux, lignes électriques, aqueducs et égouts. Le canal Lachine a été pendant un siècle le coeur industriel du Canada et les vestiges des usines et installations riveraines sont très éloquentes de la gloire passée de cette artère industrielle.

L'industrie a aussi donné naissance à des quartiers résidentiels, des artères commerciales, des equipements récréatifs. Des quartiers entiers sont nés de l'industrie: Maisonneuve à Montréal, la paroisse Sainte-Cécile à Trois-Rivières, la Basse-Ville de Québec (le bâtiment de la Dominion Corset, boulevard Charest, demeure un des joyaux del'architecture industrielledu début du siècle). Plusieurs villes du Québec sont entièrement issues de l'industrie manufacturière: Sorel-Tracy par exemple, Valleyfield, Coaticook, Hull et, bien sûr, Shawinigan.

C'est tout cet héritage que veut mettre en valeur un organisme comme l'Écomusée de la Maison du Fier-Monde dans le quartier Centre-Sud de Montréal. Cet organisme à but non-lucratif regroupe des résidents et des employés et retraités des nombreuses industries duquartier. L'Écomusée aide ces personnes à retrouver leur propre histoire, monte des expositions et organise des visites guidées d'un quartier industriel très riche. C'est une façon exceptionnelle de connaître la ville industrielle. C'est une forme de tourisme qui attire à la fois les habitants du quartier, les résidents d'autres secteurs de la ville et même des touristes étrangers. La formule s'apparente aux visites pédestres qui permettent de découvrir l'intimité de plusieurs villes: on peut faire de telles visites tant à Londres qu'à Québec.

\section{Un tourisme à développer}

L'histoire industrielle est uneécole précieuse qui attire des clientèles de toutes provenances et de tous types: écoliers, amateurs de machines et de technologie, professionnels de l'histoire sociale, amants de l'architecture.

Les sites industriels anciens qui ont été mis en valeur à des fins touristiques l'ont été grâce à l'engagement et l'ingéniosité d'individus tenaces. Ces sites posent en effet des problèmes parfois difficiles: résistance des propriétaires, détérioration avancée des bâtiments, disparition de l'appareillage, ou au contraire, richesse et importance des vestiges, taille et comple- xité des installations à mettre en valeur, manque d'expertise tant pour la connaissance que pour le développement des sites, pauvreté desmoyens financiers. Laplupart des initiatives ont néanmoins profité de subventions de la partde l'État. Toutes font appel à l'aide đu secteur privé sous forme de commandites ou à des campagnes de financementauprès du grand public de leurs régions. Certaines génèrent des revenus d'appoint par la vente de produits: farine au moulin de Saint-Eustache, articles de laine à Ulverton, papier à la papeterie SaintGilles (Charlevoix),

L'Association québécoise pour le patrimoine industriel ( $A Q P D$ ) regroupe les personnes et les groupes qui s'intéressent au patrimoine industriel. Au moyen de son Bulletin, des congrès et des visites qu'elle organise, I'AQPI permet leséchanges entre spécialistes et informe les résidents et les décideurs des régions où elle se manifeste. L'Association donne aussi aux gouvernements un médium pour saisir la problématique de la protection et de la mise en valeur du patrimoine industriel. $L^{\prime} A Q P I$ s'est intéressée à divers aspects de ce patrimoine: archives et inventaires, gestion des objets et des sites. Ses futurs congrès et Bulletins traiteront du développement urbain, de l'histoire ouvrière et (pourquoi pas?) de tourisme. $f$ 